\title{
Optimizing Outcome in Stanford Type A Aortic Dissection- A 10 Year Analysis Focusing on Surgical Techniques and Neurological Outcome
}

\author{
Frank Harig1*, Anna Engel², Johannes Rösch ${ }^{1}$ and Michael Weyand ${ }^{1}$ \\ ${ }^{1}$ Department of Cardiac Surgery, University Hospital Erlangen, Germany \\ ${ }^{2}$ Department of Cardiology and Angiology, University Hospital Erlangen Germany
}

\section{Research Article \\ Volume 3 Issue 1}

Received Date: January 08, 2019

Published Date: January 29, 2019

*Corresponding author: Dr. Frank Harig, MD, PhD, Department of Cardiac Surgery, University Hospital Erlangen Friedrich-Alexander-University Erlangen-Nuremberg, Krankenhausstr 12, D-91054 Erlangen, Germany, Tel: 0049-91318537066; Email: frank.harig@uk-erlangen.de

\section{Abstract}

Background: Today, surgical repair of aortic type A dissections is routinely mandatory but some complications remain despite technical advances over several decades. The most important complications are neurological deficits that have been quantified with an incidence of up to $25 \%$ in international trials. In this study, we focused on the postoperative outcome over a ten year period after surgical repair of type A dissections. A special focus has been put on specific complications, neurological outcome and technical advances with modern surgical techniques including cannulation strategy.

Methods: A cohort of 147 patients undergoing surgery since 2004 was studied in a retrospective analysis with 10 year follow-up. Analysis was performed using Cox-proportional hazard model focusing on more than 30 variables.

Results: Survival after $1 \mathrm{y}(5,10 \mathrm{y})$ was $98 \%(88 \%, 50 \%)$. Early mortality of all patients declined to $14 \%$ in the last years. $27 \%$ were female, with a higher age than men $(+10 y$, mean, 64+10y). Gender did not influence survival. Survival probability (Log rank test) for the first postoperative year was 0.82/0.77 (female/male) for 5 years $0.70 / 0.71$, and 0.46/ 0.50 for 10 years. Preoperatively, the carotid arteries were compromised in $25 \%$ of patients. Neurological deficits were preoperatively present in $11 \%$ of patients. Of those, a percentage of one third (33\%) was reversible. Postoperatively, major and minor neurological deficits could be seen in $22 \%$ of all patients. Over the time, the incidence of postoperative complications could be reduced (from nearly $27 \%$ to $10 \%$ ). The vascular access via truncal cannulation technique showed a significant lower risk vs. all other cannulation sites $(\mathrm{p}=0.0168)$. 


\section{International Journal of Surgery \& Surgical Techniques}

Conclusions: The incidence of neurological complications could be reduced, either as reversible preoperative deficits or postoperative new complications. Neurological disorders with concomitant carotid obstructions should lead clinicians to an extensive diagnostic approach, i.e. spreading the diagnostic look towards supraaortic/brachiocephalic branches of the aorta. By establishing technical modifications and surgical advancements, we could achieve a $50 \%$ reduction of postoperative neurological deficits from nearly $20 \%$ to below $10 \%$ and also of mortality rates from nearly $30 \%$ to about $15 \%$. This is reflected by a growing number of longtime survivors. Adopting modern perfusion techniques helps reducing the incidence of those disabilities that bear an enormous subjective medical and also economic burden.

Keywords: Neurological Outcome; Aortic Dissection; Hypothermia; Surgical Techniques

\section{Introduction}

Immediate surgical repair of aortic dissections is nowadays mandatory but complications may influence the outcome severely despite technical advances over several decades [1-4]. In the acute phase, the outcome concerning survival is severely influenced by a high acute mortality of $1-2 \%$ per hour within the initial 48 hours $[5,6]$. After surviving the acute phase, the further quality of life is mainly influenced by complications like neurological deficits that have been quantified with an incidence of nearly $25 \%$ in international trials $[7,8]$. There have been many surgical and technical advances to reduce neurological damage. Although the initial trauma can mostly not be influenced, it is important to take full advantage of all technical options during the operation like hypothermia, external head cooling and optimal brain perfusion. Some older techniques like retrograde cerebral perfusion via venous vessels (vena cava superior) have been widely used (in up to $45 \%$ of patients, IRAD-trial, 2004 [7]) but have nowadays been abandoned. Deep hypothermic circulatory arrest has also been widely used ( $78 \%$ in in the 1078 pts. of the international IRAD-trial), but its use as deep hypothermia $\left(18^{\circ} \mathrm{C}\right)$ has nowadays been reserved for very complex patients. The use of the extracorporeal circulation has been very important for the surgical approach to these diseases, but there is still a broad discussion about the optimal way of getting access to the arterial vascular system. The cannulation approach in patients with aortic dissection has ever been very complex and demanding challenging the surgeon's ability to improvisation [9]. Although sometimes critically addressed, the neuro-monitoring by near-infra-red spectroscopy (NIRS) has found a wide spread use. With some caution in interpreting values, one can observe frontal brain oxygen saturation intraoperatively and the surgeon is able to adopt perfusion strategies [10]. The strategy of adopting new technical methods and modifying proven and reliable techniques is focused on the reduction of complications and on the continuous optimization of survival and quality of life of the patients. Therefore, in this study we focused on the postoperative outcome over a ten years period after surgical repair of type A dissections. A special focus has been put on neurological outcome and technical advances with modern surgical techniques.

\section{Methods}

We studied a cohort of 147 patients during a 9-year follow-up and the diagnosis of acute type a dissection undergoing emergency surgery in our institution from 2004 to 2014. Follow-up was performed by contacting the patient and the primary care physician/ practitioner to get information about the patients' clinical status. Followup completeness was $99.1 \%$. Aim of the study was the evaluation of pre-, intra- and postoperative risk factors and their influence on survival after aortic repair for aortic dissection.

\section{Statistical Analysis}

Statistical analysis was performed using Coxproportional hazard model focusing on 36 variables. Processing of data was done by a statistician using software package R (R-Foundation for statistical computing, Vienna, Austria) [11]. In order to detect the variables with the highest impact on survival and their effect on the hazard rate, a two-step procedure was applied. In a first step, the statistical method called "boosting" was applied in order to select a subset of variables with highest impact for survival $[12,13]$. "Boosting" is a method for the selection of variables in the 
case of dealing with a high number of potential risk factors. In a second step, a Cox- Proportional -Hazardsmodel was processed using the selected risk factors in further detail [14]. This analysis was applied in the cohort of female and male patients in order to detect possible differences as described elsewhere [15]. The definition of "hazard rate":

The Hazard rate $\lambda(t)$ is defined as the risk to die in the next moment if having survived until now. The definition of "hazard ratio (HR)": HR is a ratio of hazard rates, i.e. patients with and without a risk factor:

\section{$\mathrm{HR}=\underline{\mathrm{l}(\mathrm{t}) \text { with risk factor }}$ \\ $\mathrm{l}(\mathrm{t}) \mathrm{w} / \mathrm{o}$ risk factor}

\section{Results}

\section{Operative Techniques}

The spectrum of surgical procedures comprised supracoronary aortic root replacement, (57\%, mostly using commissural resuspension of the aortic valve), Bentall's procedure (39\%), and valve sparing procedures (Repair/Yacoub's/David's procedures) in (9\%).

Additional procedures on the aortic arch and brachiocephalic vessels comprised hemiarch and partial arch replacement (18\%), complete arch repair with resection and repair of the brachiocephalic vessels (10\%), coronary artery bypass grafting (16\%). The elephant trunk and frozen elephant techniques were used in later years only to a limited extent (3.4\%).

\section{Survival}

As shown in figure 1 , the survival rate increased stepwise to an actual rate between $86 \%$ and $85 \%$ (in 2013, one third of all pts. were treated). Out of 147 patients, $6 \%(n=9)$ died in the OR, among them 7 male (7\%, of them 2 cardiogenic and 5 hemorrhagic shocks) and 2 female (5\%, 2 cardiogenic shocks).

\begin{tabular}{|c|c|c|c|c|}
\hline Surgical procedures & all & male & female & $\mathbf{P}$ \\
\hline & $147(100 \%)$ & $100(68 \%)$ & $47(32 \%)$ & n.s. \\
\hline Supracoronary AAR & $84(57 \%)$ & $58(58 \%)$ & $26(55 \%)$ & n.s. \\
\hline Infracoronary AAR & $58(39 \%)$ & $39(39 \%)$ & $19(42 \%)$ & n.s. \\
\hline Valve sparing & $13(9 \%)$ & $10(10 \%)$ & $3(6 \%)$ & n.s. \\
\hline Complete arch replacement & $15(10 \%)$ & $13(13 \%)$ & $2(4 \%)$ & n.s. \\
\hline Partial arch + hemiarch & $27(18 \%)$ & $20(20 \%)$ & $7(15 \%)$ & n.s. \\
\hline + CABG & $24(16 \%)$ & $17(17 \%)$ & $7(15 \%)$ & n.s. \\
\hline (frozen) elephant trunk & $5(3.4 \%)$ & $4(4 \%)$ & $1(2 \%)$ & n.s. \\
\hline
\end{tabular}

Table 1: Surgical procedures.

Legend: Values are presented as N (\%); AAR: Ascending aortic replacement; CABG: Coronary Artery Bypass Grafting; n.s., not significant we could not find differences between the genders concerning the spectrum of surgical procedures (Table 1).

We had to suffer a loss of 39 patients (in house deaths). Out of these patients 27\% ( $n=29)$ were male and $25 \%$ $(n=10)$ female. The most frequent reasons for death were multi-organ failure due to sepsis (28\%), cardiac failure $(23 \%)$ and irreversible brain damage $(21 \%)$ without any significant emphasis on gender (Table 2). Survival increased from about 71\% (2004-2007) to about 75\% (2008-2010) to about 85\% (2011-2014). The actual data (survival rate between 86 and $85 \%$ ) comprise $31 \%$ of our patients and they are comparable to the international data (between 88 and $87 \%$ ). 


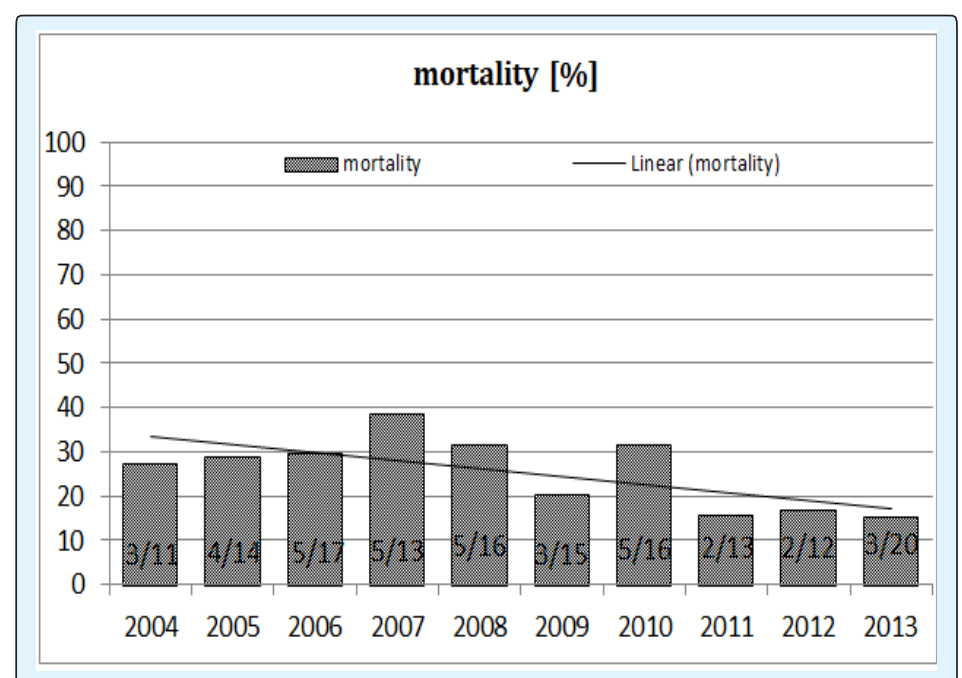

Figure 1: Mortality.

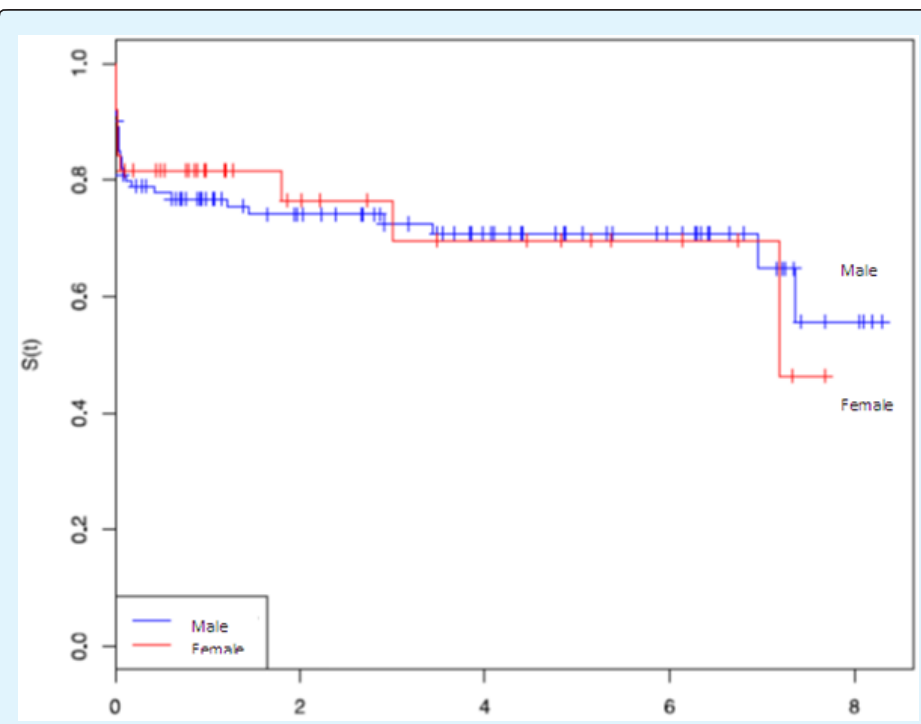

Figure 2: Kaplan-Meier- estimation for survival stratified for gender.

Legend: Cumulative Survival $\mathrm{S}(\mathrm{t})$ over the time; blue line: men; red line: women

* Taken from: Harig F, et al. [15].
The follow-up rate was 99\%. Survival declined continuously and after 1,5 , and 10 years it was 98\%, $88 \%$, and $50 \%$ respectively. The survival probability (logrank test) for the first postoperative year was $82 \%$ vs. $77 \%$ (female: male) for 5 years, $70 \%$ vs. $71 \%$, and $46 \%$ vs. $50 \%$ for 10 years. Log-rank-Test for cumulative survival could not show a significant gender specific difference $(\mathrm{P}=0.964)$ (Figure 2). These data were presented previously in a gender-focused presentation [15]. They give useful additional information in this context.

\section{The Role of Cardiovascular Risk Factors}

In the pathophysiology of cardiovascular as well as cerebrovascular diseases, the role of hypertension is widely accepted [16-20]. As described previously [15] in this cohort, arterial hypertension was present in nearly two thirds (63\%) of all patients $(71 \%$ of women, and $60 \%$ in men). Surprisingly, more women suffered from arterial hypertension. Women had a stronger compliance than men, but treatment with antihypertensive medication was only found in $56 \%$ of all (of those, $68 \%$ were female and $51 \%$ male). The body mass index as one risk factor for hypertension, was found to be significantly higher in men $(29.8+5.9)$ than in women $(26.8+4.6, \mathrm{P}=0.001)$ (Table 2).

\section{Age Groups and Gender}

In the cohort of the 147 patients with acute Stanford Type A dissection, the proportion between men and women was $3: 1$. Nearly one fourth $(27 \%)$ of patients were female, and nearly three fourth $(73 \%)$ were male. Women were 10 years older $(64+/-10 y)$ than men $(53.4+/-12 y)$ $(\mathrm{P}=0.03)$. In the 8th decade of life, percentage of women was threefold higher as in men ( $38 \%$ vs. $13 \%$ men) whereas in the 5th decade, percentage of women was only one third as in men (32\% vs. $12 \%)$. Figure 1 as mentioned elsewhere [15]. Interestingly, a higher age was not a risk factor for survival in women, but a risk factor for survival in men $(10 \%$ more risk of mortality for 10 more years of age) (Table 2) [15]. 


\begin{tabular}{|c|c|c|c|c|}
\hline & Overall & male & female & $p$ \\
\hline \multicolumn{5}{|l|}{ Demographic data } \\
\hline $\mathrm{n}[\%]$ & $147(100 \%)$ & $107(73 \%)$ & $40(27 \%)$ & $\ldots$ \\
\hline age [years], & $56.4(12.8)$ & $53.4(12.6)$ & $64.4(9.5)$ & .003 \\
\hline Body mass index [BMI] & $28.9(5.7)$ & $29.8(5.9)$ & $26.8(4.6)$ & .001 \\
\hline History of hypertension & 87 (59\%) & $60(56 \%)$ & $27(67.5 \%)$ & n.s. \\
\hline \multicolumn{5}{|c|}{ Preop. clinical signs and presentations } \\
\hline Resuscitation & $4(2.7 \%)$ & $4(3.7 \%)$ & 0 & n.s. \\
\hline myocardial infarction & $6(4.0 \%)$ & $4(3.7 \%)$ & $2(5 \%)$ & n.s. \\
\hline Cardiogenic shock/ catecholamines & $13(8.8 \%)$ & $10(9.3 \%)$ & $3(7.5 \%)$ & n.s. \\
\hline EuroSCORE (logistic) & $31.4(17.7)$ & $28.6(15.6)$ & $38.6(29.8)$ & .03 \\
\hline Aortic valve compromise & $64(43.5 \%)$ & $49(45.8 \%)$ & $15(37.5 \%)$ & n.s. \\
\hline Carotid arteries compromise & $34(23.1 \%)$ & $24(22.4 \%)$ & $10(25 \%)$ & n.s. \\
\hline LV-function, $\mathrm{EF}>50 \%$ & $83(91 \%)$ & $59(92 \%)$ & $24(89 \%)$ & n.s. \\
\hline Neurological only pre-op. deficits & $16(10.9 \%)$ & $12(11 \%)$ & $4(10 \%)$ & \\
\hline Hemiparesis & $13(8.8 \%)$ & $9(8.4 \%)$ & $4(10 \%)$ & \\
\hline \multicolumn{5}{|c|}{ Clinical and surgical data } \\
\hline OP-time & $331(112)$ & $335(116)$ & $322(102)$ & n.s. \\
\hline Time on pump/ ECC-time & $181(81)$ & $180(83)$ & $181(78)$ & n.s. \\
\hline Cross-clamp time & $99.4(48)$ & $99.9(49)$ & $97.8(47)$ & n.s. \\
\hline ICU- stay & $7.1(11.2)$ & $7.4(12.4)$ & $6.3(6.9)$ & n.s. \\
\hline Hospital stay & $16.1(13.3)$ & $16.2(14)$ & $16(11.3)$ & n.s. \\
\hline Complications and mortality" & $n=138$ & $n=100$ & $n=38$ & \\
\hline Bleeding* & $36(26 \%)$ & $24(24 \%)$ & $12(32 \%)$ & n.s. \\
\hline Reexploration: bleeding/tamponade** & $23(17 \%)$ & $16(16 \%)$ & $7(18 \%)$ & n.s. \\
\hline Pneumonia & $39(28 \%)$ & $30(30 \%)$ & $9(23 \%)$ & .06 \\
\hline Neurol. deficits (all-type) pre+post-op & $33 / 147(22 \%)$ & $24 / 107(22 \%)$ & 9/40(23\%) & n.s. \\
\hline Only post-op deficits & $17(11.6 \%)$ & $12(12 \%)$ & $5(13 \%)$ & n.s. \\
\hline Hemiparesis & $13(9 \%)$ & $8(8 \%)$ & $5(13 \%)$ & n.s. \\
\hline Intracerebral bleeding & $3(2.2 \%)$ & $2(2 \%)$ & $1(2.6 \%)$ & n.s. \\
\hline Mortality - in hospital (2005-2014) & $39(26.5 \%)$ & $29(27.1 \%)$ & $10 / 40(25 \%)$ & n.s. \\
\hline - in hospital (2011-2014) & $7 / 47(14.9 \%)$ & $5 / 47(10.6 \%)$ & $2 / 47(4.3 \%)$ & n.s. \\
\hline Operative mortality & $9 / 147(6.1 \%)$ & $7 / 107(6.5 \%)$ & $2 / 40(5 \%)$ & n.s. \\
\hline
\end{tabular}

Table 2: Demographics and clinical data of patients by gender* 
Legend: Values are $\mathrm{n}(\%)$ or mean $+\mathrm{SD} ;{ }^{*} \mathrm{p}<0.05$, n.s.: not significant, Euro SCORE: European System for Cardiac Operative Risk Evaluation [21]; ECC: Extracorporeal Circulation; IABP: Intraaortic balloon counter pulsation, ECMO: Extracorporeal Membrane Oxygenation; ICU: Intensive Care Unit

\#only survivors ( $n=138$, male, $n=100$, female, $n=38$ ) for complications; for mortality, all pts. ( $n=147$, male, $n=107$, female, $\mathrm{n}=47$ )

*Bleeding was defined as drainage volume over $500 \mathrm{~mL}$ and below $1000 \mathrm{~mL}$, which did not require re exploration (trigger was $200 \mathrm{~mL}$ per hour, or $1000 \mathrm{~mL}$ in sum)

** Reexploration was done if drainage volume was $>1000 \mathrm{~mL}$ with normal coagulation lab values within the first postop. $12 \mathrm{~h}$

*partially taken from: [15].

\section{The Incidence of Neurological Deficits}

All- type neurological deficits occurred in $22 \%$ (33/147). But there could be observed some patients with a preoperative deficit that could not be observed postoperatively. For this reason, we analyzed the only preoperative neurological focal deficits that occurred in $11 \%(n=16 / 147)$ of all patients without a main emphasis on gender (Table 2). This means that these patients had a reversible neurological deficit.

A compromise of carotid arteries, caused for example by a true lumen collapse was found in nearly every fourth patient (23.1\%), equally distributed between the genders. Due to this perfusion restriction, the most common major clinical sign was a hemiparesis (all: $13 / 147=8.8 \%$, male: $9 / 107=8.4 \%$, female: $4 / 40=10 \%$ ) (Table 2 ). As partially mentioned elsewhere [15] the preoperative cardio circulatory condition was very critical (resuscitation, cardiogenic shock, myocardial infarction) in about $15 \%$ of patients (this differs to previous studies $[7,8]$, where one third of patients were classified as 'high-risk'). This may explain a neurological deficit per se as a hypoxic brain damage due to low oxygen delivery.

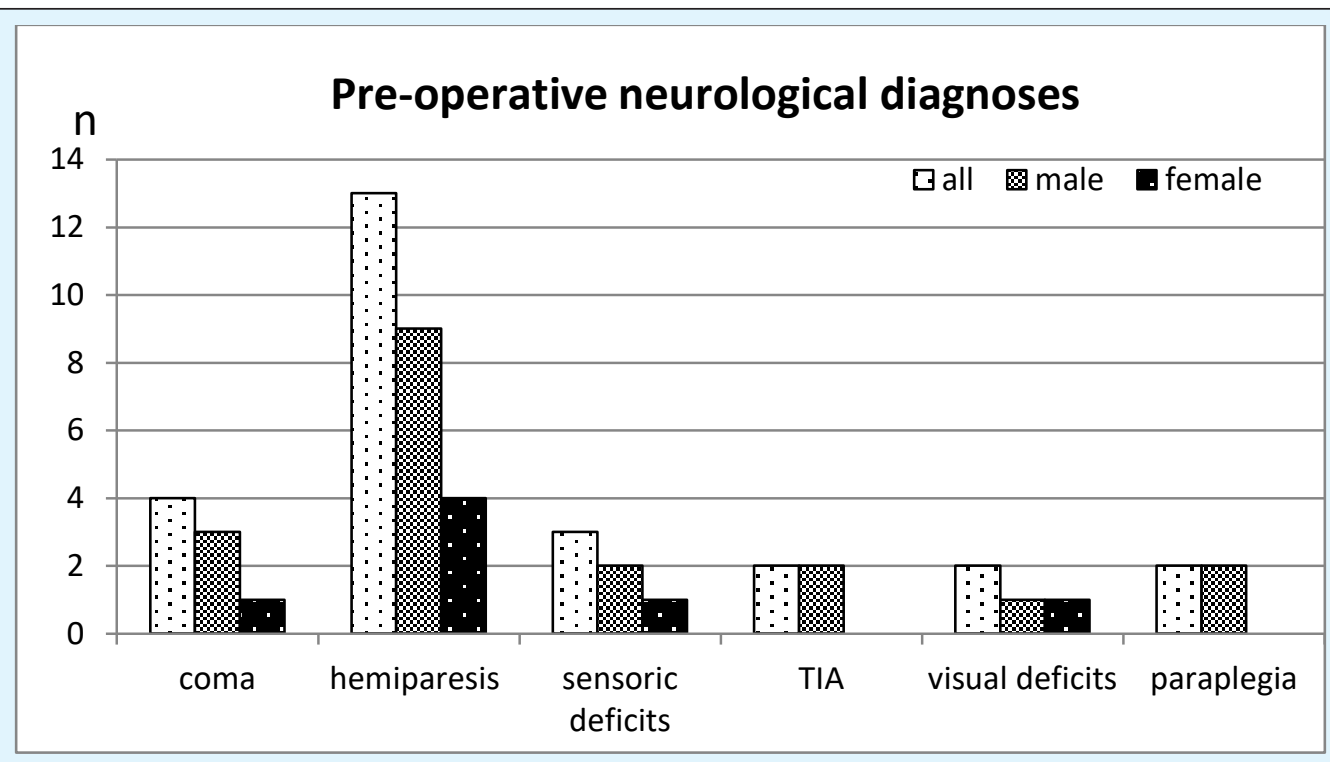

Figure 3: The clinical neurological signs of patient's ad admission.

Legend: Only descriptive clinical diagnoses are given. Values are absolute numbers. For relative values, please see text.

The neurological status at admission showed various clinical signs and we could observe a wide spectrum of deficits. The incidence of only preoperative neurological deficits varies over the years about $10.9 \%$ (range: $0-$ 23.1).

\section{Postoperative Neurological Deficits}

The incidence of any-type pre- and postoperative neurological deficits has been $22 \%(33 / 147)$ in all patients, $23 \%$ in female pts. $(9 / 40)$ and $22 \%$ in men 


\section{International Journal of Surgery \& Surgical Techniques}

(24/107), this was not significant $(\mathrm{P}=0.7671)$. The neurological deficits that were preoperatively not clinically apparent, the only-postoperative deficits, were $12 \%(17 / 138)$, in male $12 \%(12 / 100)$ and $13 \%(5 / 38)$ in female patients. Most common major deficits were stroke or hemiplegia, in $9 \%$ of all patients (in $8 \%$ of men and $13 \%$ of women, n.s., $\mathrm{P}=0.767)$. An intracerebral bleeding was only rarely the cause of major deficits $(2 \%$ men vs. $2.6 \%$ women). Spinal cord ischemia due to dissection was found in $7 \%$ of men $(7 / 100)$, but not in women (Figure 4)

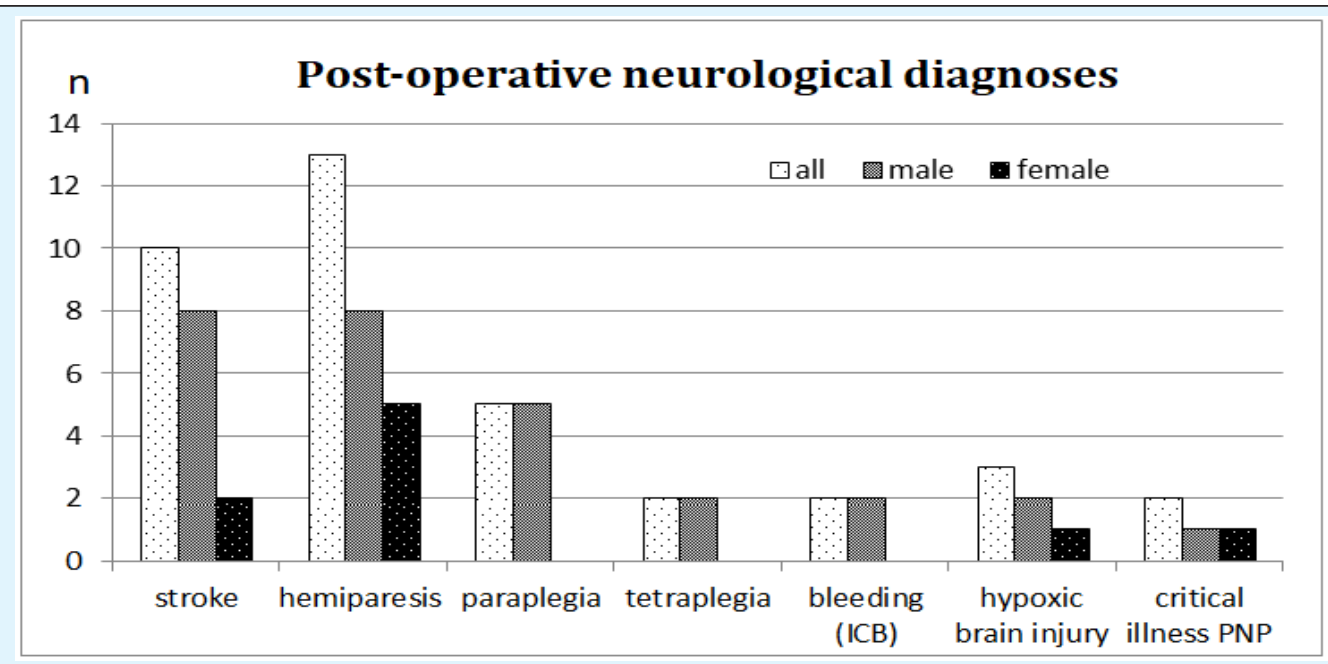

Figure 4: The clinical neurological signs of patients postoperatively.

Legend: Only descriptive clinical diagnoses are given. Values are absolute numbers. For relative values, please see text.

The incidence of postoperative neurological complications could be reduced over the time (linearly from $27 \%$ to $10 \%$ ).

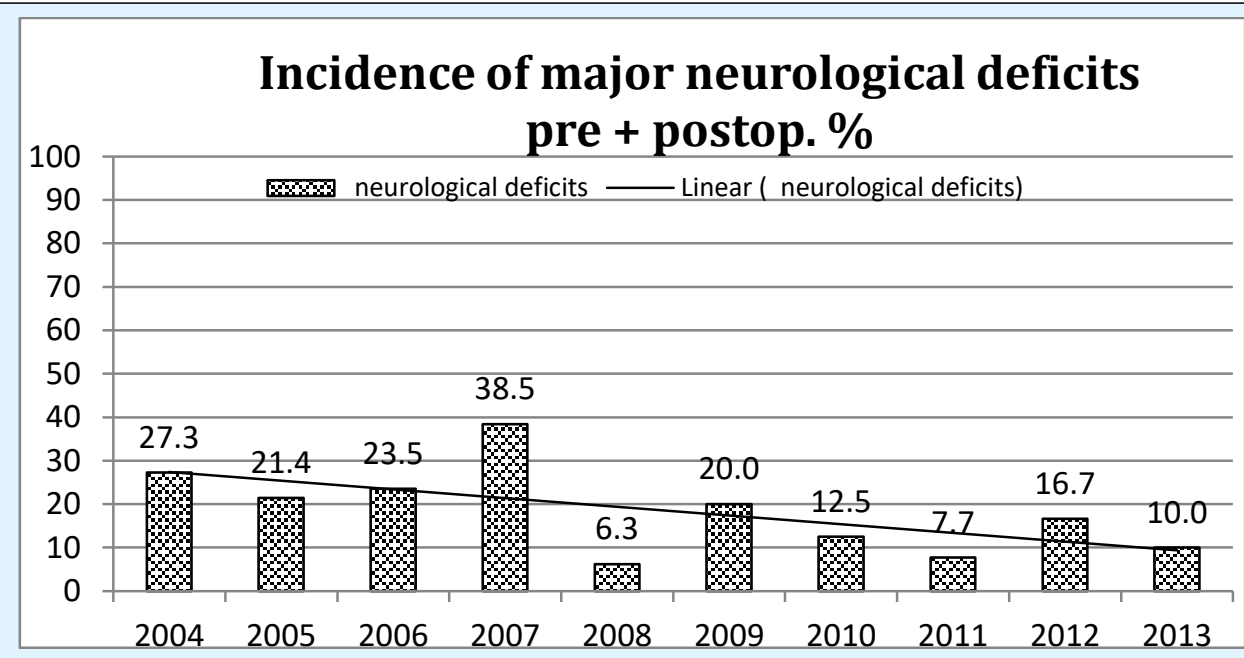

Figure 5: The incidence of major neurological deficits over the years.

Legend:

*2007: additional information:

$5 / 13$ pts. with neurol. deficits:

3 pts. suffered from global hypoxic brain damage (preop.intubated)

2 pts. suffered from paraplegia/ tetraplegia 


\section{International Journal of Surgery \& Surgical Techniques}

\section{The Cannulation Site for Establishing ECC and}

In our up to 9 year follow- up study, we could observe an overall neurological/ cerebrovascular event rate (major and minor) of $21.8 \%$, major event rate was, $17.7 \%$. Different cannulation sites were divided into 5 groups (A to E). The results are shown in table 3 . The cannulation sites that were mostly chosen were subclavian and innominate artery (74\%), femoral (14\%) and aortic arch (10\%). In group A (cannulation of the innominate artery/

\section{the Influence on Neurologic Outcome}

truncus brachiocephalicus, directly or via graft), an incidence of $19.8 \%$ was observed. These patient subgroup was most extensive and comprised nearly two thirds $(64 \%, n=91 / 147)$ of our patients. We compared group A $(n=94)$ to all others (sum of B, C, D, E, $n=53$, because each of them were too small). A significant higher incidence of neurological complications (37\%) compared to group A $(\mathrm{P}=0.0168)$ could be found.

\begin{tabular}{|c|c|c|c|c|}
\hline Arterial cannulation site & $\mathbf{n}(\mathbf{\%})$ & Let. & Neurol. Complications \# & P \\
\hline A: Innominate/ & $94(64 \%)$ & $3(2.0 \%)$ & $\mathbf{1 8 / 9 1 ( 1 9 . 8 \% )}$ & $0.0168^{*}$ \\
\hline B: Femoral & $20(14 \%)$ & $1(0.7 \%)$ & $9 / 19(47.4 \%)$ & \\
\hline C: Axillary/ & $15(10 \%)$ & $1(0.7 \%)$ & $4 / 14(28.6 \%)$ & \\
\hline D: Aortic arch & $14(10 \%)$ & $3(2.0 \%)$ & $3 / 11(27.3 \%)$ & \\
\hline E: Carotid & $3(2 \%)$ & $1(0.7 \%)$ & $1 / 2(50 \%)$ & \\
\hline B+C+D+E & $53 / 147(36 \%)$ & $6 / 53(11.3 \%)$ & $17 / 46(37 \%)$ & \\
\hline All & $147(100 \%)$ & $9(6.1 \%)$ & $35 / 138(25.4 \%)$ & \\
\hline
\end{tabular}

Table 3: The arterial cannulation site and the influence on neurologic outcome.

Legend: \#major (stroke, hemiparesis) and minor (TIA) neurol. complications

${ }^{*} \mathrm{P}$ testing: A vs. all others (B,C,D,E) because of the distribution, the main group A was tested against all others, sum of B C D E.

It seems that anatomical and pathophysiological considerations concerning blood flow in acute type A dissection might have a high impact for the patients. Femoral cannulation was only used in case of emergent installation of extracorporeal circulation. It is considered as high risk option because of retrograde flow and the high immanent risk of perfusing the false lumen without a safe cerebral or other organ perfusion [15].
The relation between the incidence of certain vascular access sites and neurological complications is shown in figure 6 . The high volume access site 'truncus' shows the lowest incidence of neurological complications, whereas the carotid and aortic arch access show a high incidence of neurological complications. 


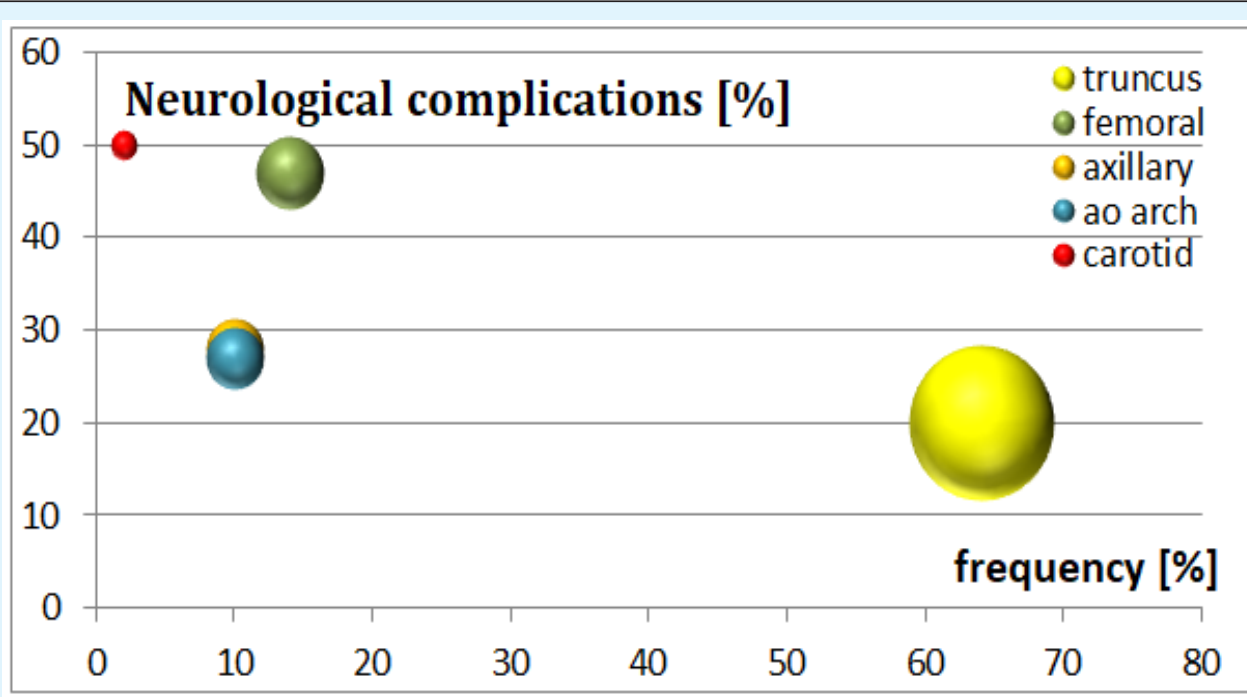

Figure 6: The relationship between the incidence of neurological complications and the frequency of the use of a special cannulation site.

Legend: The ballon histogram shows a small balloon correlating to small numbers and the greater one correlating to big ones. The vertical position indicates the frequency of neurological complications. In the case of truncal cannulation, the big balloon indicates the most frequent use and the lowest vertical position indicates the lowest complication rate.

The use of different cannulation sites has changed over the years. The use of subclavian/axillary artery was changed towards a more frequent use of the innominate artery that has become the favorite access to the arterial vascular system. Other arteries like femoral, carotid or the aortic direct cannulation were used in the case of dissection of the innominate or in the case of high urgent situations (severe circulatory depression due to tamponade, circulatory arrest) (Figure 7). In sum, over the last years, the femoral cannulation was only used in high urgent cases without other options for arterial cannulation. Central cannulation sites like innominate artery or axillary/subclavian arteries are preferred over the last years.

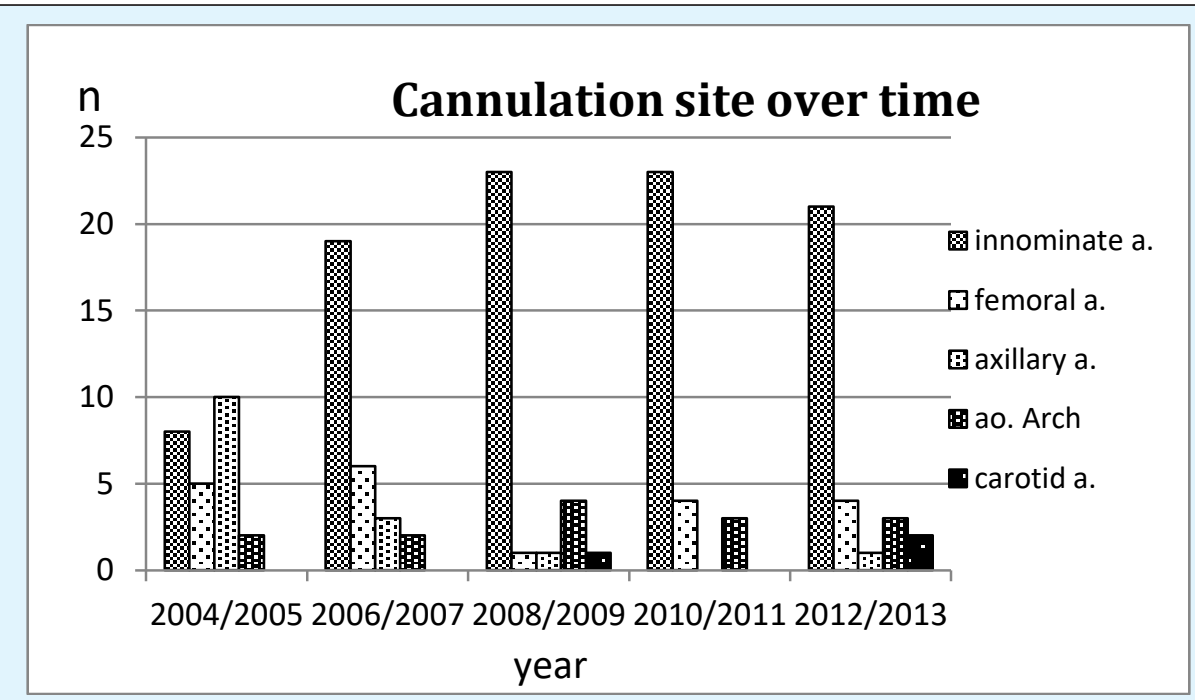

Figure 7: The distribution of the cannulation sites over the years.

Legend: Values are given in absolute numbers. Two following years are presented in each group. 


\section{The Incidence of Neurological Complications}

As shown in figure 8, the incidence of only postoperatively detectable complications over the time could be reduced from $18 \%$ to $5 \%$. This means, that a neurological deficit could not be diagnosed preoperatively, potentially due a later clinical manifestation of an earlier initiated trauma or cerebrovascular accident. We suppose that the interaction of new or additional techniques could be responsible to achieve a constant improvement over the time.

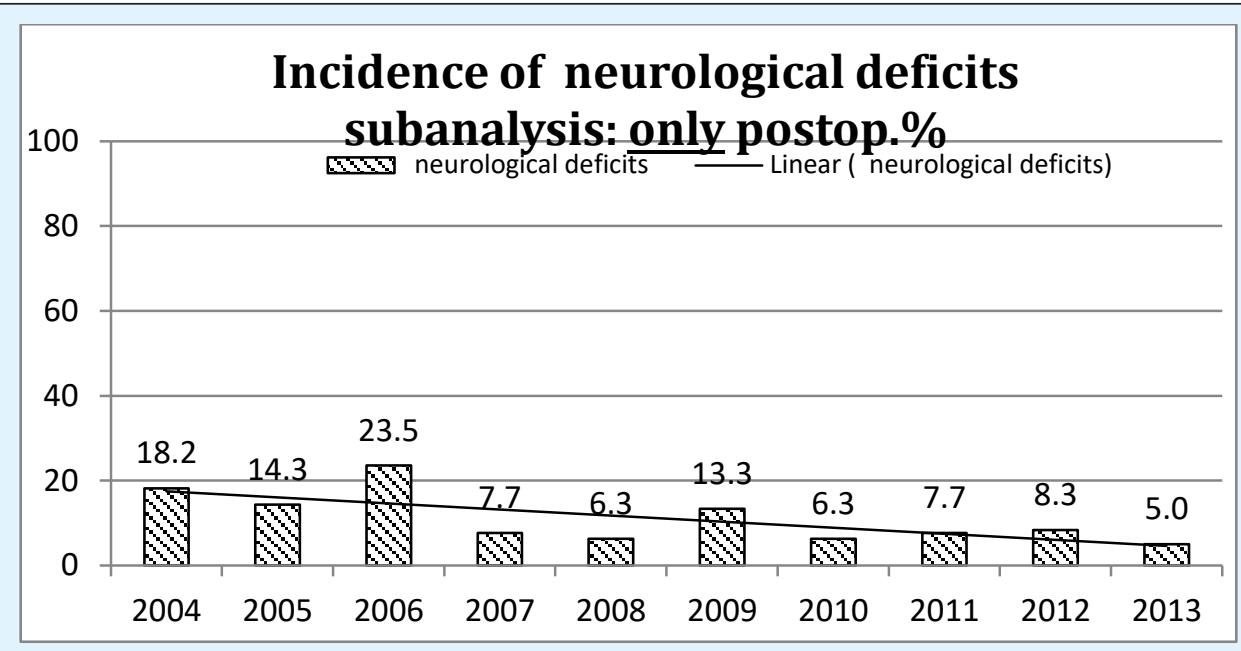

Figure 8: The incidence of neurological complications over the time.

Legend: Values are given in [\%], "only postoperative" means that the neurological status changed toward worse with a neglect that could not be seen pre- but only postoperatively.

The incidence of neurological deficits disappearing postoperatively (although they could be clinically observed preoperatively) increased over the years. This means that initially observed neurological deficits could not be diagnosed postoperatively. We consider this a change to better (Figure 9), because technical improvements could help reducing initially existing neurological deficits due to malperfusion and hypoxia.

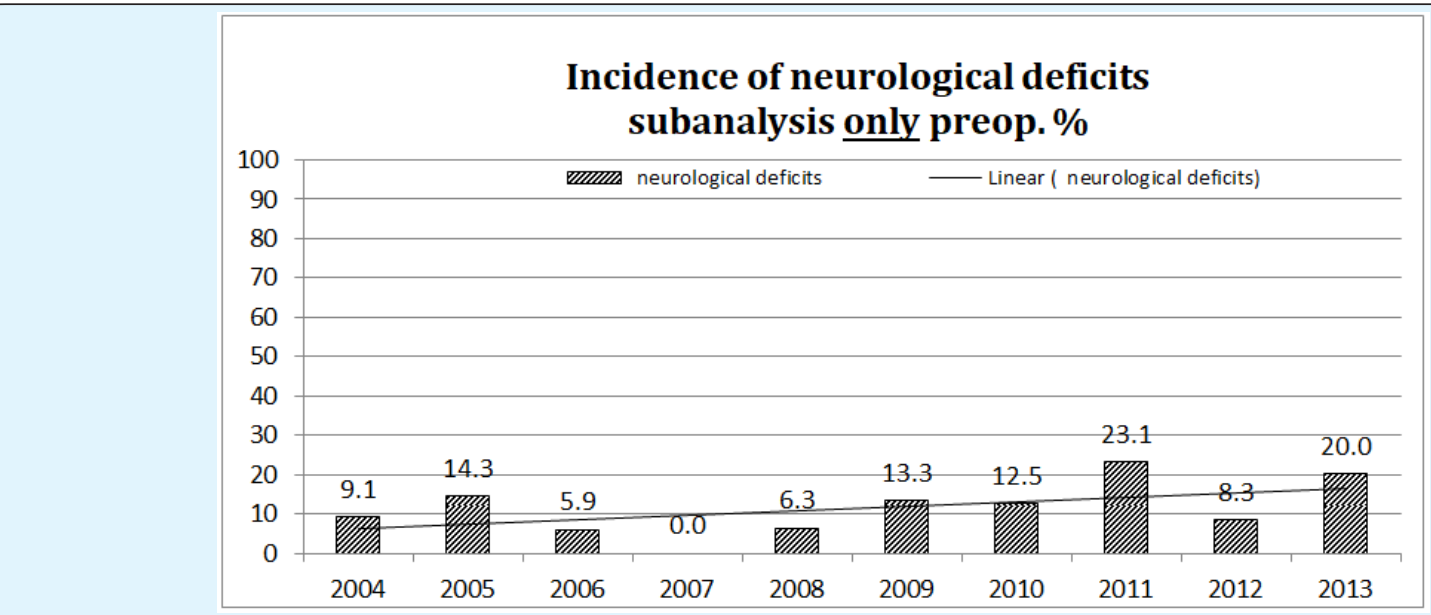

Figure 9: The incidence of only preoperative (reversible) deficits.

Legend: Values are given in [\%], "only preoperative" means that the deficits were reversible with no neglect postoperatively. 


\section{Additive Perfusion Techniques}

Monitoring the brain perfusion: Simultaneously, we used cerebral oximetry (near infra-red spectroscopy, NIRS; INVOS ${ }^{\mathrm{TM}}$ 5100C (Medtronic/Covidien, Minneapolis, MN, USA) since 2006 and FORE-Sight ${ }^{\mathrm{TM}}$ (CAS Medical Systems Inc., Branford, CT, USA) since 2010 in order to monitor frontal lobe CNS oxygen saturation that gives us a parameter of sufficient frontal brain perfusion [22].

Adopting the body temperature management: We could adopt the temperature management to a moderate extent (MHCA, $28^{\circ} \mathrm{C}$ ) without giving up the safety of former techniques. The lowest body temperature (mostly measured in the bladder by a sensor-tipped catheter) of about $16^{\circ} \mathrm{C}$ (mean $24^{\circ} \mathrm{C}$ ) since 2004 were stepwise increased up to $28^{\circ} \mathrm{C}$ (mean $30^{\circ} \mathrm{C}$ ) in recent years (Figure $10)$. During this time, the cerebral perfusion techniques were intensified, so that a decrease of neurological complications could be observed without negative side effects of deep hypothermic cardio circulatory arrest.

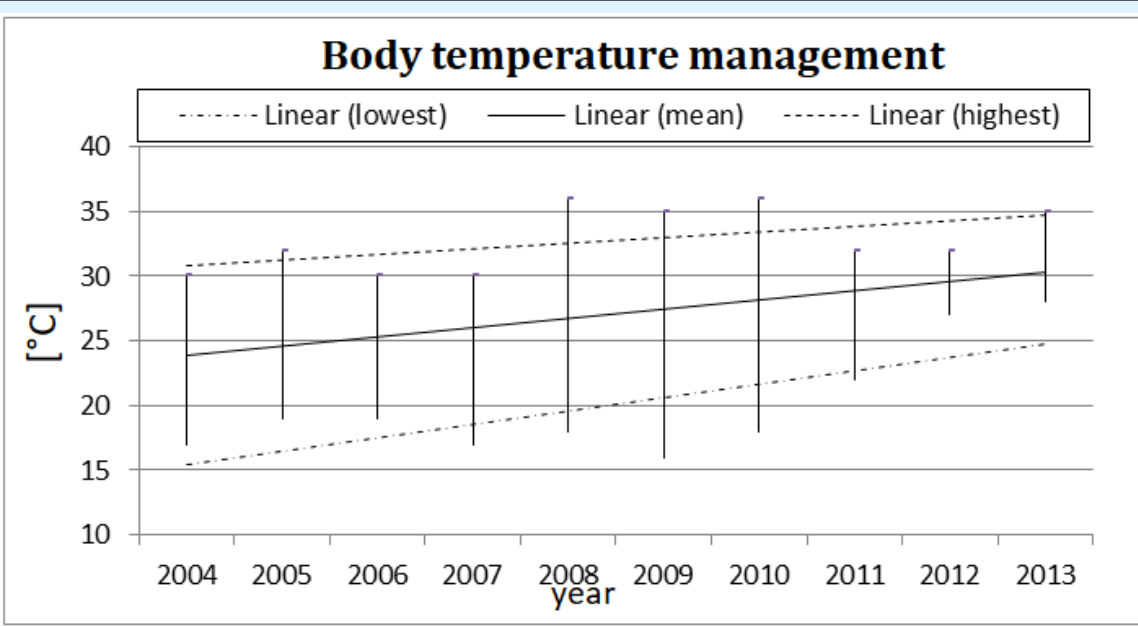

Figure 10: Temperature management.

Legend: Vertical lines indicate the maximum and minimum of body temperature for each year. Horizontal lines indicate mean values.

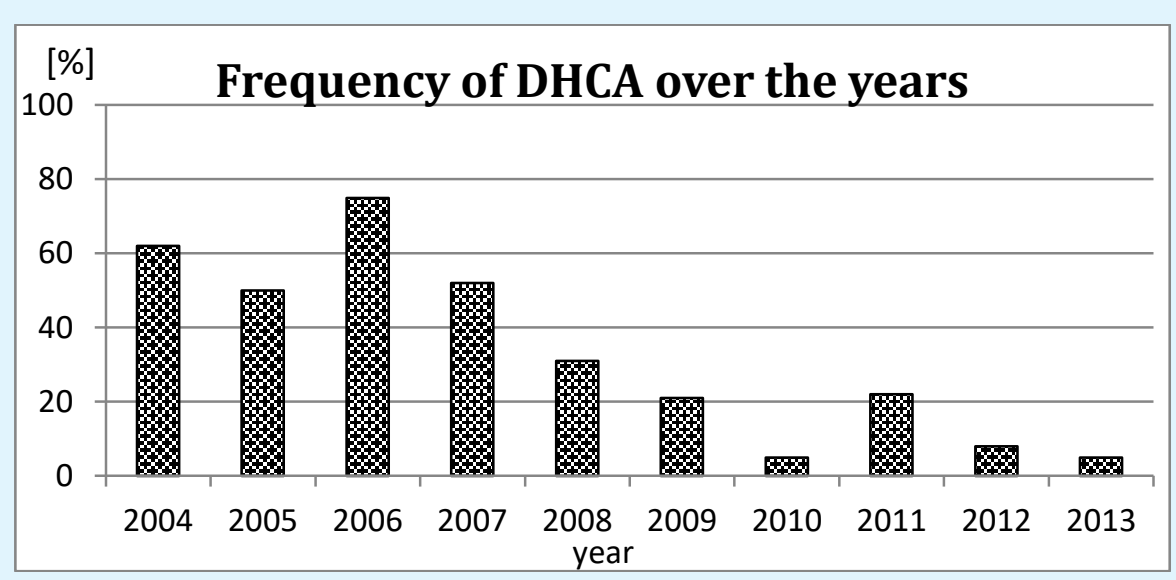

Figure 11: Frequency of deep hypothermic circulatory arrest (DHCA).

Legend: DHCA, deep hypothermic cardiocirculatory arrest: temperature below $20^{\circ} \mathrm{C}$.

The frequency of deep hypothermic cardiocirculatory arrest, that means a complete arrest when the 


\section{International Journal of Surgery \& Surgical Techniques}

temperature of $18^{\circ} \mathrm{C}$ is reached, has been reduced over the years. Whereas $50-75 \%$ of patients were deeply cooled down in the years from 2004 to 2007, in the last years, DHCA was a technique that has only rarely been used (about 5\%).

\section{Risk Factor Analysis}

A risk factor analysis in this cohort was already addressed previously [15]. In brief, scoring systems like the EURO-Score [21] try to give an objective evaluation of the preoperative clinical status. According to the EUROScore, women were admitted into hospital in a clinically severely compromised status (ES: 38 vs. 28 in men, $\mathrm{P}=0.03)$. The risk factor analysis revealed that women have a high hazard ratio for death in case of resternotomy (OR 16.543), bleeding (OR 8.1), renal insufficiency (OR 3.4). The EURO-Score showed an OR of 1.103, 95\% CI, 1.01 to $1.21, \mathrm{P}=0.038$ ) and the length of hospital stay as an indicator of survival (OR 0.849, 95\% CI, 0.74 to 0.97 , $\mathrm{P}=0.015$ ) were significant.

This means that each additional unit of EURO-Score, that reflects the severity of clinical status, leads to a 1.1fold higher hazard rate, i.e. 10\% higher mortality risk. The length of stay shows a reciprocal effect, i.e. every further day leads to a reduction of the hazard rate of $15 \%$.

Male patients had a high hazard ratio for resternotomy (OR 6.7), apoplexy (OR 2.1), renal insufficiency (OR 1.5). Only resternotomy (OR $6.7,95 \%$ CI 2.65 to 16.94 , $\mathrm{P}<0.001$ ) and age (OR 1.05, 95\% CI 1.0 to $1.1, \mathrm{P}=0.034$ ) reached levels of significance. This means, that with each year of age, the hazard rate grows 1.05 -fold, so that a 70 years old patient bears a 10.5-fold higher hazard rate (for mortality) compared to a 60 years old patient (with otherwise identical risk factors) [15].

\section{Discussion}

\section{How to Overcome Diagnostic Difficulties}

The clinical presentation of a patient suffering from a dissected aorta can be diverse. This variance of clinical signs creates the association of treating a 'chameleon'. The recognition of the underlying disease is often difficult for the initially treating physician. A trauma surgeon or orthopedist, for example, will be inclined to interpret an initial heavy acute back pain as an acute syndrome of the spinal column, e.g. lumbo- ischialgia. The neurologist in the emergency room will easily interpret an epileptic seizure due to an ischemic cerebral insult as the consequence of a carotid artery occlusion but will probably not consider the underlying disease of an aortic dissection and therefore he will probably not extend the angiogram to the brachiocephalic arteries or even to the aorta. Even the vascular surgeon getting presented a patient with an ischemic limb will not obligatory think of a dissection, but the angiogram as a diagnostic tool of the aorta will rapidly show the underlying disease without delay.

\section{Survival First, but the Quality of Life is Later on in the Center of Interest}

The initial survival rate in our patient cohort increased stepwise to an actual rate between 86\% and 85\% in 2013. This is comparable to other reported international data (88-87\%) [7,23-25]. The follow-up rate was 99\%. Survival declined continuously and after 1,5 , and 10 years it was $98 \%, 88 \%$, and $50 \%$ respectively. The survival probability (log-rank test) for the first postoperative year was $82 \%$ vs. $77 \%$ (female: male) for 5 years, $70 \%$ vs. $71 \%$, and $46 \%$ vs. $50 \%$ for 10 years. (see figure 2, taken from [15]). Logrank-Test for cumulative survival could not show a significant gender specific difference $(\mathrm{P}=0.964)$ [15]. In the case of surving the initial 'vascular accident' and further on the complex aortic repair, the quality of life is considerably determined by the neurologic status preand postoperatively. Therefore, a systematic review of outcome data is recommended.

\section{The Heart and the Brain}

The incidence of only pre-operative neurological deficits has been $11 \%$ without an emphasis on gender. The most common major clinical sign was a hemiparesis (8.8\%). The risk factor analysis shows a higher hazard ratio for male patients for apoplexy (OR 2.1), but this did not reach levels of significance [15], (Table 2). All only postoperative neurological deficits could be observed with an incidence of $12 \%$. Considering all minor and major neurological deficits, pre- and postoperatively, we could observe an incidence of $22 \%$ without an emphasis on gender. In other studies like the IRAD-trial [7], neurological deficits have been quantified with nearly $25 \%$, among them, focal neurological deficits occurred in $14 \%$, coma and altered consciousness in $10 \%$ of all patients. Hiratzka, et al. [26] described an incidence of neurological deficits from 17\% (type A and B aortic dissections) to $29 \%$ (only type A). Ischemic Stroke occurred in $53 \%$ of those patients (predominantly right hemispheric) and 37\% having ischemic neuropathy (limb pain with sensory or motor deficit). Paraplegia occurred 


\section{International Journal of Surgery \& Surgical Techniques}

in 1 to $3 \%$ of patients, comparable to our data $(1.4 \%)$. It is of special interest whether preoperatively presented deficits are still present after the operation. Hiratzka, et al. [26] reported of $50 \%$ reversible deficits. We could observe a percentage of one third (33\%) of reversible deficits. With other words, in $67 \%$ of patients, preoperative deficits persisted.

\section{The Decision for the Best Cannulation Site}

The surgical repair of a Stanford type A aortic dissection requires the use of an extracorporeal circulation (ECC) and the installation of this ECC demands an adequate arterial and venous cannulation. The optimal cannulation strategy remains a challenge for the surgeon. From anatomical aspects, there exist various cannulation techniques, for example the central (e.g. aortic) and peripheral sites (e.g. femoral artery, axillary artery or truncus brachiocephalicus/ innominate artery). From hemodynamic aspects, an antegrade flow to the brain is the most efficient option to prevent embolic atheromatous stroke or other organ malperfusion. In the case of an emergency situation, the femoral arteries provide an easy access to the circulation and may be used as cannulation sites for an immediate installation of the ECC.

But this option of retrograde perfusion carries a high risk of false lumen perfusion and of inducing embolic dislocation of atheromatous debris to other organs. We prefer the innominate artery (either direct or via vascular prosthesis) because there is no need for an extra incision and preparation of the subclavian/axillary vessels. Especially in obese patients, who are concerned over average, this vascular access may be time consuming. After sternotomy and before opening the pericardium, usually the innominate artery is prepared and an instant access to the arterial system is installed. The use of a graft (8mm vascular prosthesis), enables us to perfuse the brain antegrade while the truncus brachiocephalicus is cross-clamped in the phase of open aortic arch repair. In the case of direct cannulation, the cannula has to be redirected towards the head as described by Zielezinski et al. [27].

The cannulation of the innominate artery offers the possibility of antegrade cerebral perfusion during aortic arch surgery. The unilateral cerebral perfusion can be completed by bilateral antegrade cerebral perfusion via a left carotid artery catheter in the case of open arch reconstruction. In the case of femoral cannulation, there are some risks of false lumen perfusion and embolizing debris or atheromatous material towards the brain. A flap may obstruct the ostia of the brachiocephalic branches or other vital organs. In a meta-analysis of 14 studies, Tiwari, et al. [28] could show neurological defects in up to $17 \%$ of patients. In our study, we could see in $22 \%$ of patients all-type (minor and major) neurological defects (Table 2). In the sub-analysis over time, we could observe a reduction in neurologic deficits over the time from about 25 to about 10\% (see trend line, Figure 5) and a 9 to $18 \%$ increase in reversible deficits (see trend line, figure 9) Summarizing the literature concerning the vascular access, the anatomic location of cannulating is determined by the individual extension of the dissection, the patient's cardio circulatory stability and the individual adoption of the surgeon's abilities.

\section{Adopting of Modern Perfusion and Monitoring Techniques}

The use of modern perfusion techniques with a neuroprotective impact enabled us to modify other standard techniques like deep hypothermic cardiocirculatory arrest (DHCA, $18^{\circ} \mathrm{C}$ ). This technique has been used for years because of the organ protective effect of hypothermia. But negative side effects on the coagulation and the complement system as well as a long operative time could be observed and play an important pathophysiologic role for further complications during the postoperative course.

\section{Monitoring the brain perfusion}

Simultaneously, we used cerebral oximetry (near infrared spectroscopy, NIRS; INVOSTM 5100C (Medtronic/Covidien, Minneapolis, MN, USA) since 2006 and FORE-SIGHT ${ }^{\mathrm{TM}}$ (CAS Medical Systems Inc., Branford, CT, USA) since 2010 in order to monitor frontal lobe CNS oxygen saturation that gives us a parameter of sufficient frontal brain perfusion [29]. Although the NIRS technique had to face some critical comments, the FORE-SOGHTTM. technology has some technical advances as it utilizes five wavelengths of light, and a sensor design that allows the near-infrared light to penetrate up to 25 percent deeper than other devices. Of course, there is no device being able to monitor the global central nervous system thus lacking complete cerebral tissue surveillance.

\section{Adopting the Body Temperature Management}

Over years, deep hypothermic cardiocirculatory arrest (DHCA, $18^{\circ} \mathrm{C}$ ) was the standard technique in order to protect organs by reducing the tissue oxygen demand. We could adopt the temperature management to a moderate 


\section{International Journal of Surgery \& Surgical Techniques}

extent (MHCA, $28^{\circ} \mathrm{C}$ ) without giving up the safety of former techniques. During the last years, the cerebral perfusion techniques were intensified, so that a decrease of neurological complications could be observed without negative side effects of deep hypothermic cardiocirculatory arrest, that has been used only rarely in very severe cases.

\section{Conclusion}

The complications of an acute aortic type A dissection are severe, so that an immediate surgical approach is a life-saving strategy. Early and late mortality can be reduced by adopting and modifying new surgical techniques. Technical modifications can lead to better outcome data, for example brain monitoring and protection by NIRS, optimized cannulation techniques and temperature management are important elements. In our observational study, we could see a considerably reduction of early and late mortality and a constant increase of long time survivors. The incidence of neurological complications could be reduced, either as reversible preoperative deficits or postoperative new complications.

Neurological deficits occur preoperatively to a certain percentage ( $11 \%$ in our 10y survey) without an emphasis on gender, although male patients showed a higher Odds Ratio for apoplexy (OR 2.1) without reaching level of significance. A compromise of carotid arteries is observed frequently (25\%). This observation should lead physicians being faced with neurological disorders with concomitant carotid obstructions to a more extensive diagnostic approach, i.e. spreading the diagnostic look towards supraaortic/ brachiocephalic branches of the aorta in order find the correct diagnosis more quickly. By establishing technical modifications and surgical advancements, we could achieve a 50\% reduction of postoperative neurological deficits from nearly $20 \%$ to below $10 \%$ and also of mortality rates from nearly $30 \%$ to about $15 \%$. This is reflected by a growing number of longtime survivors. Adopting modern perfusion techniques helps reducing the incidence of those disabilities that bear an enormous subjective medical and also economic burden.

\section{References}

1. DeBakey M, Cooley D, Creech O (1955) Surgical considerations of dissecting aneurysms of the aorta. Ann Surg 142(4): 586-612.
2. Wheat MW (1980) Acute dissecting aneurysms of the aorta: diagnosis and treatment. Am Heart J 99(3): 373-387.

3. Saunders KB, Bentall HH (1967) Aneurysm of the aortic root with gross aortic incompetence: successful surgical correction. Proc R Soc Med 60(8): 726-728.

4. David TE, Feindel CM (1992) An aortic valve-sparing operation for patients with aortic incompetence and aneurysm of the ascending aorta. J Thorac Cardiovasc Surg 103(4): 617-621.

5. Kouchoukos NT, Dougenis D (1997) Surgery of the thoracic aorta. N Engl J Med 336: 1876-1889.

6. Mehta RH, Suzuki T, Hagan PG, Bossone E, Gilon D, et al. (2002) Predicting in-hospital mortality in acute type A aortic dissection. Circulation 105: 200-206.

7. Hagan PG, Nienaber CA, Isselbacher EM, Bruckman D, Karavite DJ, et al. (2000) The International Registry of Acute Aortic Dissection (IRAD): new insights into an old disease. JAMA 283(7): 897-903.

8. Nienaber CA, Fattori R, Mechta RH, Richartz BM, Evangelista A, et al. (2004)on Behalf of the IRAD. Gender-Related Differences in Acute Aortic Dissection. Circulation 109(24): 3014-3021.

9. Sabashnikov A, Heinen S, Deppe AC, Zeriouh $\mathrm{M}$, Weyamnn A, et al. (2016) Axillar or Aortic Cannulatioon for Aortic Repair in Patients with Stanford A dissection? Ann Thor Surg 102(3): 787795.

10. Rubio A, Hakami L, Münch F, Tandler R, Harig F, et al. (2008) Noninvasive control of adequate cerebral oxygenation during low-flow antegrade selective cerebral perfusion on adults and infants in the aortic arch surgery. J Card Surg 23(5): 474-479.

11. R Core Team (2013) A language and environment for statistical computing. R Foundation for Statistical Computing, Vienna, Austria.

12. Buhlmann P, Hothorn T (2007) Boosting Algorithms: Regularization, Prediction and Model Fitting. Statistical Science 22(4): 477-505.

13. https://cran.rproject.org/web/packages/mboost/vignettes/mboos t.pdf 


\section{International Journal of Surgery \& Surgical Techniques}

14. Klein JP, Moeschberger ML (1998) Survival Analysis. Techniques for Censored and Truncated Data, 2nd printing, springer, New York.

15. Harig F, Engel A, Rösch J, Weyand M (2018) A GenderFocused Analysis of 36 Perioperative Risk Factors on Long Term Survival of Acute Type A Aortic Dissection - Equal Chances? World J Surg Surgical Res 1: 1075.

16. Wenger NK, Speroff L (1993) Packard B. Cardiovascular health and disease in women. N Engl J Med 329(4): 247-256.

17. Marrugat J, Sala J, Masia R, Pavesi M, Sanz G, et al. (1998) Mortality differences between men and women following first myocardial infarction. JAMA 280 (16): 1405-1409.

18. Malacrida R, Genoni M, Maggioni AP, Spataro V, Parish S, et al. A (1998) comparison of early outcome of acute myocardial infarction in women and men. $\mathrm{N}$ Engl J Med 338(1): 8-14.

19. Vaccarino V, Parsons L, Every NR, Barron HV, Krumholz HM (1999) Sex-based differences in early mortality after myocardial infarction. N Engl J Med 341(4): 217-225.

20. Meinertz T, Diegeler A, Stiller B, Fleck E, Heinemann MK, et al. (2015) German Heart Report 2013. J Thorac Cardiovasc Surg 63(2): 86-96.

21. Roques F, Michel P, Goldstone AR, Nashef SA (2003) The logistic Euro SCORE. Eur Heart J 24(9): 881-882.

22. Bickler PE, Feiner JR, Eilers H, Rollins M (2011) Performance of 5 cerebral oximeters during hypoxia in healthy volunteers. Proceedings of the 2011 Annual Meeting of the American Society of Anesthesiologists.

23. Sabashnikov A, Heinen S, Deppe AC, Zeriouh $M$, Weymann A, et al. (2017) Impact of gender on longterm outcomes after surgical repair for acute Stanford A aortic dissection: a propensity score matched analysis. Interact Cardio Vasc Thorac Surg 24(5): 702 707.
24. Berretta P, Patel HJ, Gleason TG, Sundt TM, Myrmal T, et al. (2016) IRAD experiences on surgical type A acute dissection patients: Results and predictors of mortality. Ann Cardiothorac Surg 5(4): 346-51.

25. Erbel R, Alfonso F, Boileau C, Dirsch O, Eber B, et al. (2001) Diagnosis and management of acute aortic dissection: recommendations of Task Force on Aortic Dissection, European Society of Cardiology. Eur Heart J 22(18): 1642-1681.

26. Hiratzka LF, Bakris GL, Beckman JA, Bersin RM, Carr $\begin{array}{llll}\mathrm{VF} & \text { et } & \text { al. } & \text { (2010) } \\ \end{array}$ ACCF/AHA/AATS/ACR/ASA/SCA/SCAI/SIR/STS/SV $M$ Guidelines for the diagnosis and management of patients with thoracic aortic disease: a report of the American College of Cardiology Foundation/American Heart Association Task Force on Practice Guidelines, American Association for Thoracic Surgery, American College of Radiology, American Stroke Association, Society of Cardiovascular Anesthesiologists, Society for Cardiovascular Angiography and Interventions, Society of Interventional Radiology, Society of Thoracic Surgeons, and Society for Vascular Medicine (developed in collaboration with the American College of Emergency Physicians). J Am Coll Cardiol 121(13): e266-369.

27. Zielezinski T, Harig F, Ballazhi F, Weyand M (2011) Cannulation of the innominate artery for surgery of acute thoracic aortic aneurysm (surgical movie). Thorac cardiovasc Surg 2011: 59-VI6.

28. Tiwari KK, Murzi M, Bevilacqua S, Glauber M (2010) Which cannulation (ascending aortic or peripheral arterial cannulation) is better for acute type a aortic dissection surgery? Interact Cardiovasc Thorac Surg 10(5): 797-802.

29. MacLeod D, Ikeda K, Cheng C, Shaw A (2013) Validation of the next generation FORE-SIGHT Elite Tissue Oximeter for adult cerebral tissue oxygen saturation. Anesth Analg 116(SCA): 1-182. 